\title{
Gestion des ressources forestières au Mali et
} revendication des territoires

Le rapport à l'autochtonie ou les migrants du bois du Mandé

\section{Faty Mbodj}

\section{(QpenEdition \\ Journals}

Édition électronique

URL : http://journals.openedition.org/anthropodev/467

DOI : 10.4000/anthropodev.467

ISSN : 2553-1719

Éditeur

APAD - Association pour l'anthropologie du changement social et du développement

Édition imprimée

Date de publication : 1 avril 2014

Pagination : 43-67

ISBN : 979-10-93476-00-1

ISSN : 2276-2019

\section{Référence électronique}

Faty Mbodj, «Gestion des ressources forestières au Mali et revendication des territoires », Anthropologie \& développement [En ligne], 37-38-39 | 2014, mis en ligne le 01 décembre 2016, consulté le 01 mai 2019. URL : http://journals.openedition.org/anthropodev/467 ; DOI : 10.4000/ anthropodev.467

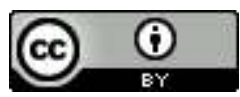

La revue Anthropologie \& développement est mise à disposition selon les termes de la Licence Creative Commons Attribution 4.0 International. 


\title{
Gestion des ressources forestières au Mali et revendication des territoires
}

\author{
Le rapport à l'autochtonie ${ }^{1}$ ou les migrants \\ du bois du Mandé
}

Faty Mbodj

\begin{abstract}
Cet article présente l'impact qu'a pu avoir le processus de décentralisation au Mali sur la gestion des ressources forestières. Les populations locales ont retrouvé leur pouvoir de contrôle sur les ressources mais sans avoir officiellement accès aux retombées financières de leur exploitation. Un système de taxation parallèle a alors été mis en place. II s'appuie sur des critères discriminants du point de vue ethnique et géographique. L'étude du cas des migrants du bois du Mandé montre la complexité des rapports qui s'établissent entre allochtones et autochtones. Ils sont basés sur une complémentarité économique mais aussi, surtout, sur la crainte des populations autochtones de perdre le contrôle de leur territoire.
\end{abstract}

This article presents impacts of decentralization process on the management of forest resources in Mali. Local populations found their supervisory power on the resources but without having officially access to the financial repercussions of their exploitation. A parallel taxes system was then introduced. It is based on discriminating criteria from ethnical and geographical point of view. The case study of migrant wood Mandé shows the complexity of relationships between immigrants and natives. They are based on an economic complementarity but also, especially, on the fear of the natives populations to lose the control of their territory.

\footnotetext{
${ }^{1}$ Le terme " autochtonie » est ici employé au sens étymologique, en tant que premier habitant, et non au sens juridique de la déclaration sur les peuples indigènes qui se trouve dans la Convention 169 de l'OIT (Organisation Internationale du Travail).
} 


\section{Introduction}

Née de la revendication des populations de voir les pouvoirs de gestion délégués à l'échelle locale, la logique de gestion décentralisée des ressources forestières a officiellement considéré les populations riveraines dans la définition des responsabilités, au travers des textes de loi. Cette politique a été vécue par ces populations telle une première preuve de reconnaissance de leur légitimité de contrôle sur les ressources. Cette reconnaissance a été confortée concrètement par la création de marchés ruraux de bois, dans la mesure où ceux-ci peuvent être considérés comme des outils de contrôle de l'accès aux ressources.

Si les populations locales ont été prises en compte dans le partage des responsabilités, qu'en a-t-il été de leur place dans la distribution des ressources financières? Les nombreuses tensions qui ont accompagné l'expérience du mode de gestion décentralisé amènent à s'interroger sur les pratiques des acteurs afin de comprendre l'origine des multiples changements qui s'opèrent présentement.

En quoi le mode de gestion décentralisé, qui a redonné aux populations riveraines leur légitimé sur le contrôle des ressources et des territoires, suite à un long pouvoir centralisé de l'Etat, a-t-il bouleversé les rapports aux territoires ? Que signifient les différents modes de négociation instaurés par les populations locales et qui sont parallèles aux règles de taxation définies officiellement par la loi ? Que reflète leur caractère discriminatoire ? En d'autres termes, qui est légitime pour l'accès aux ressources et qui ne l'est pas, au regard des populations " autochtones " ? Comment celles-ci se positionnent-elles par rapports aux différentes instances situées au niveau supérieur, qui interviennent dans la gestion des ressources forestières ? Comment la nature de leurs rapports détermine-t-elle ceux qu'elles entretiennent avec les exploitants dits " allochtones" ? Ces derniers, que nous désignerons sous le terme de "migrants du bois du Mandé ", constituent notre présent objet d'étude.

Dans la tentative de répondre à ces différentes questions, la première partie de cet article présentera quelques éléments contextuels liés au processus de décentralisation afin de comprendre comment se répartissent les responsabilités des différents acteurs intervenant dans la gestion des ressources forestières. La deuxième partie essaiera de montrer que, 
au-delà de sa pertinence institutionnelle, ce modèle de gestion présente des incohérences au niveau financier. Celles-ci se traduisent par de profondes mutations dans les rapports aux territoires, aux " allochtones » et par la mise en place de nouvelles pratiques des acteurs locaux face aux pouvoirs situés au niveau supérieur. Ces bouleversements seront traités dans la troisième partie.

\section{Politique de gestion décentralisée des ressources forestières : quelques éléments contextuels}

Dans sa démarche d'intégrer les populations locales, la politique malienne de gestion décentralisée des ressources naturelles, et forestières en particulier, a été perçue par un grand nombre d'acteurs comme une logique de rupture par rapport aux méthodes précédentes. Elle a été d'autant plus marquante, au regard des populations riveraines des espaces forestiers, qu'elle leur a redonné leur légitimité sur le contrôle et sur la gestion des ressources grâce à la création de Structures Rurales de Gestion du Bois (SRGB).

Qui est légitime pour la gestion des ressources et des territoires dans ce contexte?

A la suite de la chute du régime dictatorial, en 1991, un nouvel air démocratique a soufflé sur le Mali. II s'est traduit notamment par une révolte des populations locales contre l'Etat et les agents forestiers, ces derniers étant considérés comme les vestiges de la page sombre de la période dictatoriale. Les populations ont parlé également de "retour du pouvoir au village " (Kassibo, cité par Gautier et al, 2004). La considération que les autorités du gouvernement de transition de 1992 ont accordée alors à leurs revendications s'est traduite par la mise en place d'un Schéma Directeur du Développement Rural (SDDR). Par ailleurs, la volonté d'intégrer les populations dans la gestion des ressources naturelles a nécessité un nouveau découpage territorial correspondant à un transfert de responsabilités. C'est dans cette optique que différentes lois ont été votées.

Il s'agit de la loi 93-008 du 11 février 1993 selon laquelle « les collectivités territoriales ont pour mission la conception, la programmation et la mise en œuvre d'actions de développement économique, social et culturel 
d'intérêt social ». En outre, la loi 95-034 du 27 janvier 1995 portant code des collectivités territoriales en République du Mali confère au Conseil communal la responsabilité de délibérer sur la protection de l'environnement et l'organisation des activités rurales et des productions agro-sylvo-pastorales. Aux yeux de la loi, cela signifie que l'échelle communale (qui regroupe plusieurs villages) est désormais retenue comme le niveau de compétence le plus adapté à la gestion des ressources naturelles. Pourtant, ce choix n'exclut pas l'échelle villageoise. La loi 95-034 le confirme dans son article 17 : "Le Conseil communal est tenu de prendre l'avis du ou des conseils de village [...] pour délibérer sur la protection de l'environnement et la gestion des ressources naturelles ". L'article 28 de la loi 95-004 s'inscrit dans cette même logique de définition des rôles des collectivités territoriales en matière de protection des ressources forestières. Elle stipule que "les collectivités territoriales décentralisées sont astreintes à prendre des mesures de protection des ressources forestières ". La loi 95-050 portant principe de constitution et de gestion du domaine forestier des collectivités territoriales considère, dans son article 21, qu'il est du devoir des collectivités territoriales d'intervenir dans l'organisation des activités forestières et d'élaborer des schémas d'aménagement du territoire incluant le domaine forestier qui sera exploité. Celui-ci doit comprendre les forêts naturelles, les reboisements et les périmètres de protection classés en leur nom ainsi que le domaine forestier immatriculé en leur nom.

A ce cadre institutionnel s'ajoute la mise en place d'outils de gestion tels les Structures Rurales de Gestion du Bois (SRGB), connues souvent sous le nom de "SRG ».

Les SRG: des outils de renforcement de la légitimité des populations locales

Parallèlement au processus de décentralisation, une réforme de la législation forestière a été entreprise par le projet Stratégie Energie Domestique (SED) (Gautier et al, op.cit). Opérationnel à partir de 1999, ce projet a été cofinancé par la Banque Mondiale et la Coopération néerlandaise pour un montant de 7,9 millions d'euros. Il comprenait deux volets qui étaient la demande et l'offre. 
La demande visait l'amélioration de l'accès à l'énergie et de son utilisation, notamment sous ses formes modernes (gaz, pétrole, électricité) pour améliorer les conditions de vie quotidienne des populations. L'offre cherchait des modalités d'approvisionnement favorisant le développement économique et ne portant pas préjudice à l'environnement, grâce à une gestion rationnelle des ressources forestières par les communautés rurales. Pour cela, le projet SED a mis en place un schéma directeur d'approvisionnement des principales villes, a procédé à la création de marchés ruraux de bois et a instauré un système de taxation différentielle.

Au niveau le plus localisé de la gestion (l'échelle villageoise), le marché rural du bois a constitué une pièce essentielle du dispositif SED (Nouvellet, 2002). Il était défini comme un lieu de vente de bois et de charbon géré par une SRG agréée par la loi 95-003 du 18 janvier 1995. Selon le SDA, « la SRG [est] composée par les résidents d'un même village ou hameau et bénéficie d'un droit opposable à un tiers sur le massif qu'elle exploite à travers le contrat ". Avec cette déclaration, les populations locales recouvraient clairement une légitimité sur le contrôle des ressources naturelles, perdu pendant des décennies. Cela signifiait qu'elles assuraient désormais la gestion et l'accès aux ressources. Mais qu'en a-t-il été de leur implication dans le partage des ressources financières ?

\section{Exploitation forestière et enjeux de la redistribution financière}

Après une dizaine d'années d'expérience environ, la gestion décentralisée des ressources forestières a occasionné d'importantes tensions entre les différents acteurs qui intervenaient dans ce processus. C'est dans les enjeux économiques et, notamment, dans le mode de redistribution des ressources financières qu'il faut chercher l'origine des bouleversements qui se sont dessinés à l'échelle villageoise.

\section{Répartition des ressources financières à travers une clé floue}

Lors de la campagne de sensibilisation qui a précédé la mise en place des marchés ruraux, l'attribution de ristournes aux SRG figurait bien dans le programme et les populations en avaient été informées. En effet, le décret 95-422/P-RM, fixant le taux et la répartition des taxes perçues à 
l'occasion de l'exploitation du bois, intégrait, en son article 6 Chapitre III, les SRG au titre de bénéficiaires de la taxe d'exploitation, autant que l'Etat central. Elles avaient droit à $40 \%$ de ristourne sur les taxes perçues en zone orientée et $60 \%$ en zone contrôlée ${ }^{2}$ (voir tableau 1 ci-dessous).

\begin{tabular}{|c|c|c|c|}
\hline Exploitation & Incontrôlée & Orientée & Contrôlée \\
\hline Structure rurale de gestion & - & $40 \%$ & $60 \%$ \\
\hline Commune rurale & $50 \%$ & $40 \%$ & $30 \%$ \\
\hline Etat & $50 \%$ & $20 \%$ & 10 \\
\hline
\end{tabular}

Tableau 1 : Taux de répartition des taxes perçues

à l'exploitation forestière (1995)

Toutefois, avant le fonctionnement effectif des collectivités décentralisées et sans que les SRG puissent être remises dans leur droit, un autre décret, celui 98-402/P- RM du 17 décembre 1998, a abrogé le premier et exclu définitivement les SRG de toute perception de ristournes. La commune, de son côté, a vu sa part réduite à la portion congrue : au lieu des $50 \%$ prévus par le premier décret, elle ne perçoit plus rien sur l'exploitation en zone incontrôlée, au lieu des $40 \%$ initialement prévus en zone orientée, elle n'en perçoit plus que $5 \%$ et, en zone contrôlée, le taux passe de $30 \%$ à $10 \%$. L'Etat, à travers ses organes et ses services, s'octroie désormais la part du lion, percevant notamment la totalité des ristournes en zones incontrôlée, soit 100 \% (voir tableau 2 ci-dessous).

${ }^{2}$ La loi 95-003 du 18/01/1995 définit trois types d'exploitation grâce à un système de taxes différentielles. L'exploitation de type " contrôlé » est faite dans une zone délimitée et aménagée suivant les règles définies par un plan de gestion. Dans cette zone le $\mathrm{m} 3$ de bois est taxé à 250FCFA et le quintal de charbon à 500FCFA. L'exploitation de type " orienté " se fait dans un massif délimité et non encore aménagé. Le $\mathrm{m} 3$ de bois y est taxé à 400FCFA et le quintal de charbon à 800FCFA. Quant à l'exploitation de type "incontrôlé ", elle porte sur une zone non délimitée et non aménagée; donc n'étant pas sous le contrôle de la SRG. Elle ne doit pas non plus approvisionner le marché de bois. La taxation y est plus élevée (800 FCFA le $\mathrm{m} 3$ de bois et 1200 FCFA le quintal de charbon). 


\begin{tabular}{|c|c|c|c|}
\hline \multirow{2}{*}{ AFFECTATIONS } & \multicolumn{3}{l}{ Quotité suivant l'origine du bois } \\
\cline { 2 - 4 } & Incontrôlée & Orientée & Contrôlée \\
\hline Budget d'Etat & $60 \%$ & $35 \%$ & $15 \%$ \\
\hline $\begin{array}{c}\text { Travaux d'aménagement et d'entretien des } \\
\text { massifs forestiers }\end{array}$ & - & $30 \%$ & $45 \%$ \\
\hline Contrôle forestier & $35 \%$ & $15 \%$ & $10 \%$ \\
\hline Communes rurales & - & $5 \%$ & $10 \%$ \\
\hline Chambres régionales d'agriculture & - & $5 \%$ & $10 \%$ \\
\hline Remises aux agents forestiers & $5 \%$ & $10 \%$ & $10 \%$ \\
\hline
\end{tabular}

Source : article 8 du décret $\mathrm{n}^{\circ}$ 98-402/P-RM du 17 décembre 1998

Tableau 2 : Taux de répartition des taxes perçues

à l'exploitation forestière (1998)

L'objectif de la part réservée à la commune, aussi réduite soit-elle, est de lui permettre de développer des projets environnementaux (plantation d'arbres, entretiens de forêts villageoises, etc.). Ceci signifie que les fonds perçus par la commune et générés par les forêts situées sur les territoires villageois doivent faire l'objet de réinvestissements environnementaux dans la mise en œuvre, par exemple, de projets de reboisement ${ }^{3}$.

Quant aux SRG, l'article 8 du décret 98-402/P-RM du 17 décembre 1998 ne les mentionne même pas; ce qui a suscité beaucoup d'incompréhensions chez les villageois qui se fiaient toujours au premier décret. Une année après la phase fonctionnelle des SRG, ces dernières n'ont rien perçu. Les gestionnaires des SRG ont alors réclamé leur dû devant le bureau communal, ce qui a engendré de sérieux problèmes (Keita, 2003). Mais les représentants communaux ont argumenté leur position en s'appuyant sur le deuxième décret qui n'indique pas clairement la part qui revient aux SRG dans la répartition des taxes perçues sur l'exploitation forestière. Ainsi, les recettes ont été versées aux collectivités alors même

${ }^{3}$ Un décret de 2004 a encore modifié la clé de répartition entre l'Etat et les collectivités locales (respectivement $80 \%, 20 \%$ ). Mais comme il confondait à la fois les différents paliers des collectivités mais également les 3 zones de taxation, il n'a pas été suivi (Cf. Antona et al., dans ce numéro). 
que les travaux d'entretien des massifs forestiers incombaient aux populations riveraines, membres des SRG.

Du fait de leur marginalisation dans la redistribution des recettes de l'exploitation, les populations villageoises ont instauré alors un système de taxation discriminatoire.

Système de taxation en marge des textes de loi : l'expression des revendications

Plus que l'expression d'une frustration liée au manque de considération économique dont elles sont victimes, le mode de taxation instauré par les populations locales est révélateur d'une forme de revendications sur les ressources et sur les territoires qui les portent. Il évoque ainsi et surtout la revendication d'une légitimité qu'elles avaient perdue devant la puissance de l'Etat centralisé.

En effet, traditionnellement, l'accès aux ressources naturelles était réglementé par des droits coutumiers à l'échelle du village. En général, les prélèvements de bois de feu étaient libres sur toutes les terres non cultivées. Sur les terres cultivées, l'activité se conformait aux systèmes de tenure en vigueur. Le droit de propriété sur la terre impliquait le droit de propriété sur les arbres (Sidikou, H.A., 1997, cité par Gazull, 2003). Seule l'autorité villageoise était habilitée à donner des autorisations pour l'exploitation forestière. Ces autorisations étaient octroyées en fonction des terres concernées, de la nature des espèces végétales présentes et de la position sociale du demandeur. Ce contrôle avait essentiellement pour objectif de préserver les ressources naturelles d'une dilapidation éventuelle par des "étrangers " au village, notamment les pasteurs, les villageois voisins ou les nouveaux arrivants (Benjaminsen, T.A., 1997, cité par Gazull, op.cit).

Historiquement, la mainmise de l'Etat sur les ressources s'est substituée à la gestion locale par les chefferies villageoises. Cela a entraîné beaucoup de frustrations chez celles-ci et chez les populations riveraines de façon générale, qui ont vu venir sur leurs territoires des bûcherons travaillant pour les commerçants urbains qui n'ont eu de cesse de 
s'enrichir grâce à la valeur commerciale de la ressource liée à l'accroissement de la demande des villes.

La logique de gestion décentralisée leur a redonné leur légitimité en leur confiant la gestion des ressources. Cependant, au niveau économique, tout portait à croire qu'elles n'étaient pas considérées. Dans leur choix $d^{\prime}$ instaurer des taxes, en marge de celles définies officiellement par les textes de loi, on peut voir plus qu'un moyen de signaler leur mécontentement aux instances territoriales et politiques situées au niveau supérieur (Etat, Direction nationale de la conservation de la nature, autorité décentralisée). Il est la preuve évidente de l'expression d'une revendication pour recouvrer un pouvoir considéré comme légitime sur les ressources et qui s'exprime par un contrôle strict de la présence d'étrangers sur le territoire. Face à ces remaniements au niveau local, le pouvoir de l'Etat, à travers les compétences des agents forestiers mais aussi à travers celui des collectivités décentralisées, semble être limité. D'une certaine manière, on même est en droit de penser que, si l'Etat ferme les yeux sur ces négociations officieuses, il semble reconnaître, par là même, leur légitimité. Aussi est-il devenu systématique pour les exploitants forestiers de payer aux villages une petite somme de 100 FCFA sur chaque sac de charbon ou 15000 FCFA à 20000 FCFA pour chaque chargement de camion. Cependant, cette taxation est discriminatoire car elle ne concerne que les exploitants étrangers aux territoires villageois.

\section{Une taxation discriminatoire : l'identité ethnique et géographique des acteurs concernés}

Les taxes fixées par les populations locales ne concernent nullement les exploitants autochtones. Elles ne visent qu'un seul groupe d'exploitants que nous appelons les " migrants du bois du Mandé ». Leur mode de désignation par les villages d'accueil ne fait pas référence à leurs origines géographiques mais plutôt à leurs appartenances professionnelles et ethniques. Ainsi sont-ils communément appelés " charbon mbara ${ }^{4}$ » ou plus

${ }^{4}$ Littéralement « les travailleurs du charbon » et donc « les charbonniers ». 
simplement " les Bambara » bien qu'il existe, parmi eux, quelques exceptions, notamment des populations dogons et malinkés ${ }^{5}$.

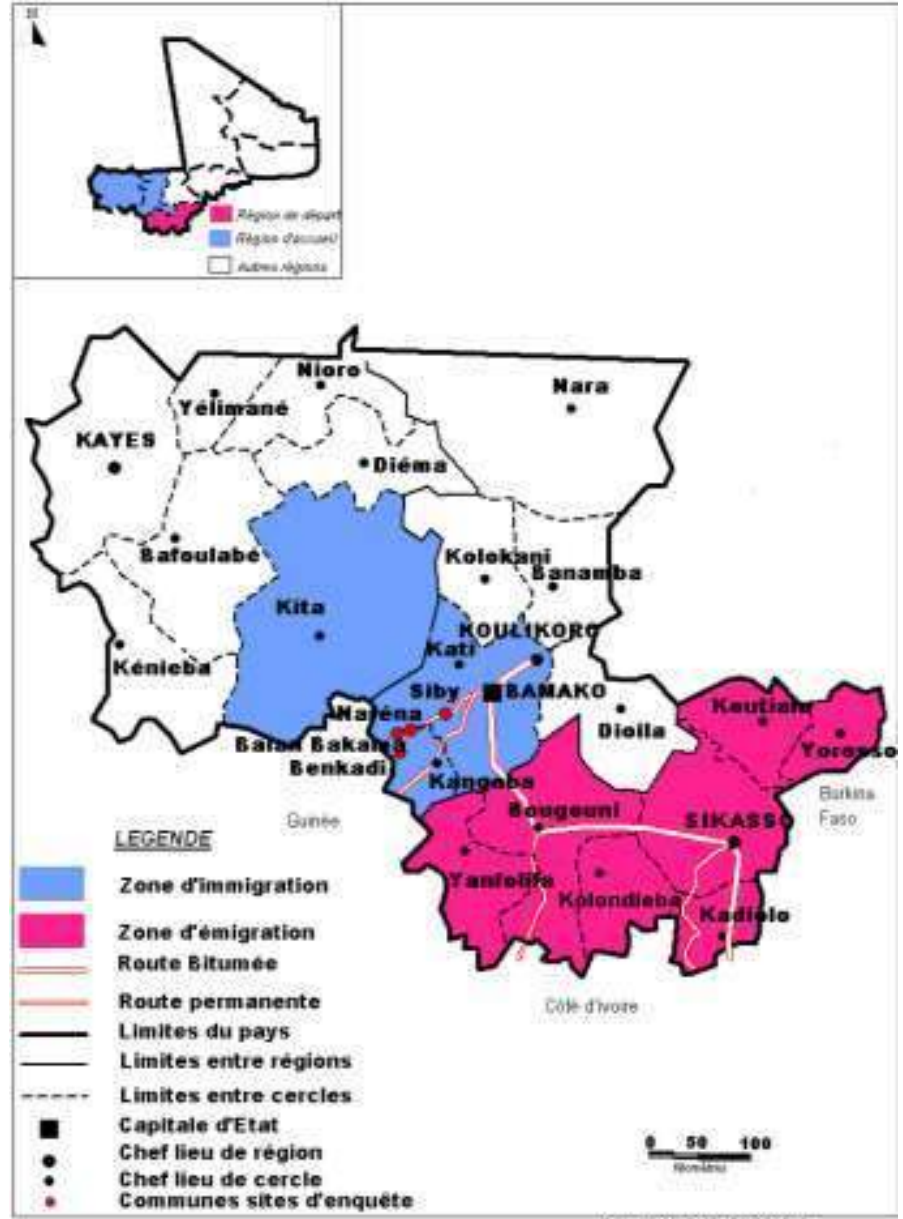

Carte

1 :

Pré-

senta-

tion

des

zones

de

migra-

tion.

5 Les Bambara ont une tradition de bûcheronnage et de carbonisation très ancienne. Alors que les Malinké, fiers de leurs origines royales (descendants du roi Soundiata Keita), se sont tardivement investis dans ce métier, et l'ont fait dans le contexte de la décentralisation. Voir Bertrand A.1992. Les résultats de ses enquêtes montrent des facteurs socio-culturels à ce type d'activité. Les Bambara restent dominants dans l'exploitation du bois autour de Bamako. 
En effet, les migrants du bois du Mandé sont originaires de la région de Sikasso et particulièrement du cercle de Bougouni (voir carte 1 ci-dessous), une zone géographique où les populations sont réputées pour leurs compétences en carbonisation - pratique dans laquelle ils se spécialisent de plus en plus au détriment de la vente de bois. La plupart d'entre eux ont hérité cette pratique de leurs parents. Ces derniers l'ayant eux-mêmes apprise en Côte d'Ivoire - très proche de Sikasso - où ils entretenaient des relations particulières via le grand nombre de ses ressortissants qui y résident et dont certains ont réussi à faire partie de puissants réseaux de commerçants de charbon.

Avant de devenir commerçants, la plupart de ceux-ci étaient d'abord des exploitants travaillant avec les planteurs ivoiriens selon un système de collaboration que l'on voit parfois chez les migrants du Mandé. Lorsque des planteurs avaient besoin de défricher des terres de culture, certains maliens, guinéens et burkinabé leur servaient de bûcherons non payés mais qui avaient un droit exclusif sur les pieds d'arbres que certains transformaient alors en charbon pour approvisionner les villes ainsi que certaines usines.

Certes, des ressortissants de la région de Sikasso exploitaient déjà dans le Mandé depuis plus de 10 ans. Mais c'est la combinaison de plusieurs facteurs contemporains (l'accroissement de la demande en charbon de la ville de Bamako, liée à sa croissance démographique et à la mutation de la structure des ménages ; le conflit ivoirien, entrainant beaucoup de retours de jeunes à Sikasso au Mali, etc.) qui a provoqué le départ d'importantes vagues de populations jeunes de la région de Sikasso qui se sont installées dans le Mandé et qui sont spécialisées en carbonisation.

Leur installation dans ces territoires a révélé à la fois toutes les conséquences de la redistribution défaillante des ressources financières issues de l'exploitation forestière, la mutation qu'a connue le statut de ces ressources ainsi que le rapport à l'allochtonie.

\section{Territoires, migrants et populations autochtones}

D’une activité dévalorisée, peu pratiquée, l'exploitation du bois est devenue désormais une activité principale, autant que l'agriculture. Si elle n'est 
pas l'activité de tous mais de certains groupes sociaux expérimentés dans ce domaine, ces derniers ont saisi les opportunités qu'elle offre et vont jusqu'à migrer vers d'autres territoires pour la pratiquer. Toutefois, si ces groupes allochtones détiennent l'expérience professionnelle, les autochtones quant à eux, sont détenteurs de la légitimité sur le territoire et de la définition des règles d'accès aux ressources. Si, face à cette situation, les rapports de coopération seraient à privilégier afin que les deux parties puissent tirer profit du pouvoir de chacun, de nombreuses peurs imposent d'autres stratégies que chaque camp peut qualifier de minières. Elles se traduisent par des relations très instables et parfois conflictuelles.

\section{Valeur commerciale du bois : facteur de migration}

Si l'exploitation du bois a pu susciter d'importants mouvements de populations d'une région à une autre, c'est parce qu'un regard moins dévalorisant s'est porté sur cette pratique dans le contexte de décentralisation et de la montée en puissance de sa valeur marchande. Ainsi, l'évolution de la perception de cette pratique dans les pays du Sahel nous aide à comprendre les déplacements des migrants du bois du Mandé.

Quel que soit le pays en Afrique subsaharienne, le bûcheronnage et, surtout, le charbonnage sont vécus comme des activités physiquement pénibles et socialement dégradantes. Toutes les appréciations données sur cette pratique, à travers les principales enquêtes effectuées par des chercheurs auprès des producteurs dans différents pays, ont fait allusion à ces deux critères. Au Niger, il ressort que le travail de bûcheronnage reste une activité peu valorisée socialement et que ne pratiquent pas certains groupes sociaux (les pasteurs notamment) car il s'agit d'un travail physiquement dur (PEII, 1991). Au Mali, c'est la pénibilité du travail qui est mise en avant et non le niveau de rémunération. $60 \%$ des charbonniers et $40 \%$ des bûcherons pensent ne pas poursuivre longtemps l'activité parce que le travail est difficile (CCL, 1998). Au Sénégal, l'activité de charbonnage est généralement vue comme sale et dégradante. L'exploitation est majoritairement faite par des migrants d'origine guinéenne. Certains Sénégalais pensent même que l'argent du charbon est sale (Ribot.J.C., 1990, cité par Gazull, 2003). 
D'une manière générale, dans les pays du Sahel, cette activité n'était pratiquée que par les groupes castés et/ou économiquement les plus démunis, ne disposant pas de suffisamment de terres pour avoir été les derniers à s'installer dans les villages, comme l'a confirmé une étude de la CCL en 1998. Toutefois, cette donnée est à relativiser car dans le cas qui nous intéresse, les charbonniers sont venus de la région de Sikasso qui fait partie de ce que l'on appelle le "triangle de prospérité ${ }^{\text {" }}$ du Mali et qui est l'une des principales zones de production de coton et des autres cultures céréalières du pays. Par ailleurs, à quelques exceptions près, les densités de population n'y sont pas très préoccupantes.

En fait, d'une pratique très dévalorisée, l'exploitation du bois énergie est entrée dans la logique des stratégies de diversification des revenus des paysans (Brondeau, 2001). Elle permet ainsi de gérer les risques liés à une agriculture très tributaire des aléas climatiques et de la fluctuation des cours mondiaux. Pour les plus pauvres, disposant de peu de terres, elle est une protection contre la famine. Pour la majorité des ménages, elle permet également l'accès à des biens et services de commodité : habillement, dépenses sociales. Pour les plus riches, elle permet d'augmenter la productivité agricole par l'achat d'une charrette, d'engrais, d'animaux ou d'outils. Enfin, pour les mieux nantis, elle est source de revenus supplémentaires (CCL, 1998 ; Molller, 1998).

Ainsi, pour certains groupes de populations, le choix de migrer dans d'autres régions naturelles, parmi lesquelles le Mandé7, est à chercher dans la place qu'occupe désormais l'exploitation du bois dans l'économie rurale ; une place d'autant plus importante que les filières de commercialisation sont puissantes et assistent les migrants du bois, même dans leur intégration dans les territoires d'accueil.

\footnotetext{
${ }^{6}$ Ce triangle concerne Sikasso-Bamako-Mopti.

${ }^{7}$ Cette région est proche de Bamako (la distance maximale est de $80 \mathrm{~km}$ ) et dispose encore d'importantes potentialités forestières.
} 


\section{Modalités d'intégration des migrants dans les territoires du Mandé}

Pour comprendre le mode d'intégration des migrants du bois dans les territoires du Mandé, il faut d'abord analyser la structure de la filière commerciale du bois énergie et saisir les formes de sa diversification en cours.

Partout dans le Sahel, il n'existait jusque là que deux types de filières bois. L'une correspond à la forme généralisée au Niger et au Sénégal. Elle se caractérise par une certaine forme oligopolistique (Ribot, 1990) détenue par des commerçants-transporteurs sur la production primaire et sur l'approvisionnement des villes ${ }^{8}$. L'exploitation du bois est effectuée par des bûcherons salariés employés en ville et envoyés en général dans les zones à fort potentiel ligneux. La plupart de ces employés sont également des migrants en quête d'un travail. Ils sont appréciés des commerçants transporteurs qui les emploient car ils n'ont pas de liens avec les populations des villages où ils vont exploiter. Ils sont aussi très pauvres et sont ainsi très dépendants (Ribot, 1998 ; PEII, 1991). Leur employeur leur fournit des vivres, à titre d'avance remboursable sur la production, et les villageois n'ont avec eux aucune occasion d'échange. Ils n'exercent aucune autre activité, sont rémunérés à la tâche et sont soumis à un rendement minimum.

L'autre type de filière accorde un rôle important au bûcheron paysan mais sans aucun lien de dépendance. Les résultats de différentes études menées au Mali, au Niger et au Sénégal ${ }^{9}$, citées par Gazull (2003), ont montré que ce type de filière tend à se généraliser au Mali, au Tchad et dans certaines zones du Niger. Seuls les rapports entre exploitant-vendeur et commerçant-acheteur structurent les relations entre acteurs; ces rapports sont facilités par un intermédiaire qui est souvent le transporteur. Toutefois, avec le cas des migrants du bois du Mandé, on assiste à la naissance d'un troisième type de filière au Mali, que l'on peut qualifier de filière intermédiaire et qui se situe donc entre les deux autres. Ce troi-

${ }^{8} \mathrm{Cf}$. article de Boutinot L., dans ce même numéro.

9 ESMAP, 1992 ; Ribot, J.C., 1995 ; CCL, 1998 ; PEII, 1991 et Antona, et al., dans ce même numéro. 
sième type permet aux exploitants migrants d'être moins dépendants des commerçants tout en bénéficiant, grâce à eux, d'une intégration plus facile sur les territoires du Mandé.

En effet, cette nouvelle filière fonctionne selon un système à étage. A la tête, se situent les commerçants, communément appelés patrons. La plupart d'entre eux sont des Bambara originaires de la même région que les migrants. Leur rôle n'est pas uniquement d'envoyer les camions à la recherche du charbon dans le Mandé. Ils sont aussi bien présents à l'amont qu'à l'aval de la filière, une position qui leur permet de contrôler la chaîne de production depuis l'exploitation, tout en laissant les exploitants indépendants, contrairement à ce qu'avait affirmé Bertrand (1992) à travers les propos suivants : "Au Mali, le contrôle des commerçants sur la filière boisénergie ne s'exerce pas par une maîtrise directe de l'exploitation [...] ». En effet, les patrons recrutent les migrants, choisissent les villages d'accueil dans le Mandé, négocient avec les autorités traditionnelles, leur fournissent le matériel nécessaire pour l'exploitation, leur font une avance en nature et paient les permis de coupe à leur place ainsi que les taxes destinées aux autorités villageoises. Malgré tout cet investissement supplémentaire de la part des commerçants, les migrants du bois ne sont cependant pas tenus de fournir un rendement minimum. Ces modes de négociations sont révélateurs d'une concurrence des commerçants dans la filière bois.

Par ailleurs, même si l'acceptation des migrants sur les territoires d'accueil ne pose presque pas de problèmes compte tenu des intérêts économiques qu'ils apportent aux autochtones, les accords sont précaires en raison du contrôle strict que ces derniers exercent, dans le cadre de la décentralisation, sur leurs territoires et sur leurs ressources.

Rapports autochtones/migrants du bois: entre enjeux économiques et enjeux de territoires

Des autochtones partagés entre méfiance et envie de coopérer

Au cœur des rapports entre les populations autochtones du Mandé et les migrants du bois venant de la région de Sikasso, se posent deux princi- 
paux enjeux : les enjeux économiques et territoriaux. Les principaux acteurs sont les populations autochtones. Elles sont partagées entre les intérêts économiques qu'elles peuvent tirer de la présence des migrants et une volonté permanente de contrôler et rappeler le pouvoir légitime qu'elles détiennent sur les ressources et les territoires. Ainsi, le schéma de leurs rapports est identique sur tout le territoire des forêts du Mandé. Les migrants sont accueillis par les populations autochtones qui profitent de leur expérience professionnelle (en matière de carbonisation) et de leurs apports financiers, en même temps qu'ils sont l'objet d'expulsions sous la pression exercée sur les autorités villageoises par différents groupes d'acteurs, notamment les jeunes autochtones et les chasseurs. Lorsque les migrants sont expulsés d'un territoire voisin et sont à la recherche d'un autre site d'accueil, le village nouvellement sollicité les accueille dans l'intérêt de ses populations résidentes, quand bien même il est informé des motifs de leur précédent délogement. Les taxes qu'ils payent, et qui sont désormais systématiques, permettent aux villages d'accueil de financer certains ouvrages et services collectifs (construction de logements d'enseignants, entretien des forages publics, salaires d'infirmières, d'enseignants d'école communautaire ainsi que paiement des dépenses nécessaires lorsque des hôtes viennent au village).

Un autre type de profit, encore plus significatif, à la fois d'ordre professionnel et stratégique, et s'inscrivant sur le long terme, fait plutôt l'affaire des jeunes autochtones qui n'hésitent pas à côtoyer ceux qu'ils appellent les Bambara pour apprendre à fabriquer des meules de charbon. Une fois les techniques maîtrisées, ils sortent du groupe des migrants pour former un bloc d'autochtones travaillant parallèlement aux autres. Finalement, sous l'effet de la concurrence, ils peuvent constituer de véritables groupes de pression auprès des autorités traditionnelles. Ils attirent ainsi leur attention sur les transgressions des migrants par rapport aux zones de coupes vers lesquelles ils étaient orientés, aux zones interdites (bords des mares), aux espèces interdites, etc. Leur objectif, à terme, est de déloger les migrants afin de se sentir libres sur leur propre territoire.

Par ailleurs, les jeunes charbonniers autochtones ne constituent pas les seuls groupes de pression qui existent dans les territoires d'accueil. Les 
chasseurs en sont un aussi ${ }^{10}$. Leurs revendications tirent leur légitimité de la crainte de la déforestation qui entraîne la disparition du gibier et qui, de fait, compromet leurs intérêts. Ainsi, ils peuvent réussir à déloger plus d'une centaine de migrants avec leurs familles, comme nous avons pu l'observer à Faraguéro et Kouémalé où plus de 40 chasseurs se sont mobilisés et ont mis le feu à la production des charbonniers ${ }^{11}$.

\section{Schéma des processus de délogement des exploitants allochtones}

Dans le processus de délogement, différents types de négociations sont adoptés par les populations autochtones. Ils concourent tous à montrer que, malgré les profits financiers et professionnels que procure l'exploitation par des étrangers, les populations autochtones ont peur de voir les membres d'un groupe socio-identitaire s'imposer économiquement sur leurs territoires ${ }^{12}$, devenir des citoyens au même titre qu'elles, notamment par les liens de mariage, et devenir influents dans les négociations. Ces craintes définissent des rapports qui tendent à se traduire par une diversité des décisions et des moyens de pression qui sont tous révélateurs d'une volonté de contrôler la présence de ces étrangers ou de maîtriser la gestion du territoire. On peut déceler trois types de négociations. Le premier consiste à expulser tous les exploitants sans exception, même ceux d'entre eux qui ont fondé une famille sur place en épousant une autochtone, de peur que des relations très affectueuses les lient dans le temps et rendent pénible toute décision. L'autre type d'arrangement est basé sur l'instauration d'un système de quota grâce auquel une partie des migrants est remerciée alors que l'autre reste sur place mais à condition

\footnotetext{
${ }^{10} \mathrm{Cf}$. article de Keïta S., dans ce même numéro.

${ }^{11}$ Cette tentative de délogement a été difficile parce que, dans le groupe d'exploitants étrangers, se trouvait une personne née et élevée à Bougouni mais dont les parents étaient malinké et originaires du Mandé. Se réclamant de ses origines malinké et donc ayant les mêmes droits que ceux qui se disent autochtones, cette personne a incité les autres migrants à ne pas quitter le village dans la mesure où ils payaient correctement les taxes. Ce climat très tendu a porté l'affaire jusqu'au cercle de Kangaba.

12 Comme c'est le cas à Naréna où nous avons rencontré Mery, un ancien charbonnier devenu boutiquier, installé dans le village même. Avec les recettes de son exploitation, il a ouvert une boutique, puis deux autres à la tête desquelles il a placé ses deux frères. Il a aussi deux autres de ses frères qui sont charbonniers dans le village.
} 
d'associer le bûcheronnage à l'agriculture. Enfin, le troisième type de négociation consiste à inviter les migrants qui souhaitent s'installer à abandonner totalement l'exploitation afin de se convertir dans l'agriculture. Seule une minorité accepte cette dernière proposition. D'une part, parce qu'elle n'a pas de sécurisation sur les terres qu'elle cultive puisque ces terres sont toujours prêtées et peuvent être retirées du jour au lendemain. D'autre part, parce que certains exploitants se plaignent de la médiocrité des terres qui leur sont attribuées. Pour cette raison, les quelques migrants qui se soumettent à ce genre de proposition, comme c'est le cas à Kourémalé, ne le font que pour maintenir leurs véritables ambitions professionnelles : produire du charbon de bois dans le territoire d'accueil. En effet, Kourémalé se situe à la frontière entre le Mali et la Guinée. Deux villages portant le même nom existent en fait, des deux côtés de la frontière, avec la précision du pays associée afin de les identifier. Cette position géographique a permis à ceux qui sont restés sur place, à KourémaléMali, de jouer sur les frontières nationales et de négocier avec les autorités villageoises de Kourémalé-Guinée pour exploiter sur leur territoire, tout en étant hébergés par la partie malienne.

\section{Les prétextes de délogement}

Les populations autochtones semblent éprouver un sentiment d'envahissement face au nombre grandissant des migrants; un sentiment perceptible à travers des propos tels que : "Ils étaient très très nombreux avec leurs femmes et leurs enfants. Ils étaient même plus nombreux que la population villageoise [...] ". On peut considérer que ces propos sont exagérés mais ils donnent une idée de la peur des populations résidentes d'être dominées économiquement et démographiquement par ces étrangers.

Dans les rapports entre les migrants du bois et les populations autochtones apparaissent, d'une part, bien des réactions subjectives récurrentes et, somme toute, classiques, laissant dire aux Malinké que les Bambara sont destructeurs de la nature, voire qu'ils sont pervers et qu'ils amènent des prostituées dans les villages, séduisent les femmes et les enfants avec leur argent, etc., et d'autre part, des assertions plus objectivées sur les pratiques des migrants accusés de dessiner des stratégies minières. En d'autres termes, tout semble faire croire aux villageois que, sachant que le 
moment d'être délogés est inéluctable, les migrants tentent de profiter des ressources du village en exploitant le maximum et le plus rapidement possible. Cette situation a d'ailleurs pu être observée à Siby (village) où des migrants avaient reçu l'autorisation de s'installer dans une forêt (Déguéré) qui appartient au village mais qui se situe dans une zone éloignée, donc échappant à son contrôle. Profitant de l'absence de surveillance, ainsi que d'une possibilité d'évacuer le charbon jusqu'à Bamako sans passer par la route goudronnée où se situe le poste de contrôle forestier, les migrants sont passés d'une exploitation autorisée à une exploitation clandestine en invitant d'autres charbonniers sur place et en utilisant des tronçonneuses. La découverte de leur exploitation frauduleuse a provoqué une enquête sollicitée depuis le niveau ministériel ainsi que la visite du Ministre de l'Environnement sur les lieux. Cet événement, et la sensibilisation du Ministre qui lui a fait suite, a marqué le début de la stigmatisation des migrants par les populations du Mandé. Tout en reconnaissant le droit des autochtones d'accueillir des étrangers sur leurs territoires, le Ministre les a en effet incités à ne pas accepter de telles pratiques. Cette situation a servi de prétexte aux villages qui ne voulaient plus de ces étrangers. Ils en ont profité pour les remercier en invoquant comme principale raison le rythme de coupe. En effet, les migrants n'ont pas une idée des superficies déboisées pour fabriquer les sacs de charbon. Le seul mode de mesure porte sur la quantité approximative de carburant utilisée: le chiffre de 20 litres d'essence pour déboiser 2 hectares environ et fabriquer 140 à 150 sacs de charbon, est avancé par les utilisateurs des machines.

\section{Négociations échappatoires entre migrants $d u$ bois et populations autochtones}

Malgré les nombreux cas de délogement, quelques migrants ont toutefois réussi à développer des stratégies leur permettant de rester plus durablement dans les villages d'accueil. Ces stratégies sont fortement liées aux critères de choix des sites d'exploitation. Ceux-ci sont au nombre de deux : d'une part, des critères de proximité et de facilité d'accès et d'autre part, des facteurs stabilisateurs. 
Le premier cas concerne les axes routiers goudronnés ou les pistes $\mathrm{OHVN}^{13}$ (praticables) grâce auxquels la production de charbon est facilement et fréquemment acheminée. Le second cas concerne des sites géographiques très enclavés, difficiles d'accès, où les camions se rendent peu fréquemment mais où les risques d'être délogé sont, en revanche, minimes. Car ce sont des zones situées dans des dépressions spécialisées dans le maraîchage. Cette dernière activité est pratiquée par les populations locales pendant toute l'année ; également en période sèche grâce à la proximité de la nappe phréatique et l'existence de marigots. Cette activité dominante fait que les jeunes autochtones travaillent aux champs à tout moment avec leurs parents et ne trouvent pas de temps pour apprendre le métier de carbonisation auprès des migrants, les concurrencer et finalement les chasser. Ainsi, dans ces zones enclavées, comme par exemple le hameau de Daa, le métier du bois continue d'être l'apanage des migrants.

D'ailleurs, au lieu de s'adonner à des jeux de concurrence et de jalousie, migrants et paysans autochtones privilégient des pratiques complémentaires. En effet, à chaque fois que les paysans ont besoin d'aménager de nouveaux espaces pour le maraîchage, ils font appel aux charbonniers. Ces derniers ne sont pas payés mais les pieds d'arbres coupés leur appartiennent. Ces types d'arrangements, même s'ils ne sont pas toujours bénéfiques pour les charbonniers, notamment dans les cas où les espèces trouvées sur place ne fournissent pas du charbon de qualité, leur permettent cependant de gagner la confiance des populations autochtones et de s'installer durablement dans les villages d'accueil; pour le moins, avant que la zone en question devienne improductive et que les autochtones aient besoin de diversifier leurs activités économiques en s'orientant aussi vers l'exploitation et la carbonisation du bois.

${ }^{13}$ Office de la Haute Vallée du Niger. 


\section{Exploitants allochtones : source de tensions entre populations de même village}

\section{Conflits de pouvoir et d'intérêts économiques face à la présence des allochtones}

Au-delà de la stigmatisation dont ils sont victimes et qui est à considérer avec leur statut d'étrangers sur les territoires d'exploitation et par rapport aux ressources, la présence des migrants du bois dans les villages du Mandé est à l'origine de beaucoup de tensions entre habitants d'un même village. Les migrants paient des taxes aux villages d'accueil mais ces intérêts sont mal partagés. Cette situation crée des divergences qui se traduisent en tensions et parfois en conflits entre autochtones. De la même manière, des tensions peuvent naître simplement parce que leur présence sur le territoire risque de compromettre les intérêts symboliques d'autres acteurs intéressés par les ressources forestières. Mais dans tous les cas rencontrés, les populations autochtones préfèrent le départ des migrants du bois de leurs territoires, dès lors que la situation risque de s'envenimer, plutôt que de voir la cohésion sociale au sein des villages se dégrader.

Par ailleurs, en dehors des tensions engendrées par des intérêts d'ordre économique, les rapports entre migrants du bois et populations autochtones ont également soulevé la problématique de l'unité territoriale. Le positionnement des différents acteurs au cours des tensions a, en effet, invité les populations à se demander si elles se sentaient appartenir à un seul et même territoire et sous l'administration d'un pouvoir unique. Car des exploitants chassés d'un centre de village peuvent être accueillis par un hameau excentré du même village ; moins pour des raisons économiques que pour exprimer une frustration liée au monopole du pouvoir que détient le centre du village sur toutes les affaires et donc sur la gestion des ressources forestières. Nous avons pu relever ce cas à Faraguéro où, lorsque les exploitants étrangers ont été chassés de Konkani (centre du village de Faraguéro), une partie d'entre eux a été accueillie par le hameau de Kouroubala. L'explication fournie par le représentant du chef de village a été que : "Lorsque ces exploitants étrangers s'installaient dans le village, on ne nous a pas informé de leur présence. Alors ce n'est pas maintenant qu'ils ont à nous dicter ce que l'on doit faire et ce que l'on ne doit pas 
faire ». En fait, cette situation montre qu'à l'échelle villageoise, le pouvoir des " $\mathrm{Soo}^{14}$ " et des chefs concernant la gestion des ressources forestières est contesté par les « Bougouda ». Nous avons alors l'impression d'assister à un processus de fragmentation territoriale parallèlement à la décentralisation. Ce qui constitue un paradoxe, puisque celle-ci privilégie des regroupements, à travers le choix de l'échelle communale. Dans ce contexte, l'arbitrage reste difficile car l'autorité décentralisée n'a toujours pas de prérogatives sur le foncier.

\section{Différents niveaux de structuration des tensions}

Les conflits d'intérêts économiques et de pouvoir, évoqués ci-dessus, se structurent généralement à partir de trois niveaux de relations : entre autorités coutumières et SRG ; entre autorités coutumières et jeunes exploitants autochtones; entre autorités coutumières et autorités décentralisées. On est tenté de penser que ces tensions peuvent se résumer à une confrontation entre un pouvoir traditionnel, incarné par les chasseurs et les conseils de villages, et un pouvoir moderne, représenté par les jeunes exploitants autochtones et les institutions modernes telles les SRG. Mais le problème est plus complexe que ce qu'une telle classification nous donne à voir car, au sein même de ces deux types de pouvoir, se profilent des divergences d'intérêts et des tensions.

En effet, si les taxes que les migrants du bois versent sont destinées à la caisse du village où ils s'installent, celles-ci sont prises en charge par le gestionnaire du marché rural de bois. II arrive que ce dernier soit tenté de s'approprier ces ressources financières appartenant à toute la communauté villageoise. De ce fait, ne voyant pas l'intérêt économique que l'exploitation par les étrangers procure à la communauté, le conseil de village peut décider de les déloger de son territoire, comme nous avons pu l'observer dans le village de Sélénia. Ce genre de situation nous donne à voir la façon dont les exploitants allochtones sont parfois victimes des problèmes entre les autochtones eux-mêmes. "Ces Malinké ont des problèmes entre eux-mêmes. Nous, on paie les taxes qu'ils nous imposent,

${ }^{14}$ Dans le Mandé, les habitats villageois sont divisés en plusieurs entités territoriales. Chaque village comprend un centre appelé « Soo » et un ensemble de hameaux ou « Bougouda ». 
alors pourquoi ne pas aller voir la personne qui a utilisé leur argent, au lieu de se rabattre sur nous ? [... $]^{15} »$

Dans d'autres situations, ce sont les jeunes exploitants autochtones qui, après avoir maîtrisé la pratique de carbonisation, s'obstinent à affirmer leur légitimité sur les ressources en se confrontant à l'autorité coutumière. Cette situation s'est notamment produite dans un village de la commune de Siby où les jeunes ont refusé catégoriquement l'accès des migrants aux ressources forestières. Ils ont exigé que le conseil de village rompe le contrat d'exploitation qu'il avait signé avec eux. Cette demande a été rejetée car l'instance considérait que les taxes payées par les exploitants rendaient un grand service à la communauté villageoise. Les jeunes ont alors décidé de mettre le feu aux sacs de charbon des étrangers. L'affaire a dû être déposée à la gendarmerie de Siby mais elle s'est finalement réglée à l'amiable et les migrants ont quitté le village.

A Siby village, chef lieu de l'autorité décentralisée, les migrants du bois ont aussi négocié avec l'un des chefs de quartier, à l'insu des autres et du chef de village ainsi que de l'autorité décentralisée. Celui-là leur a donné l'autorisation d'exploiter dans la forêt de " Déguéré » et les taxes qu'ils payaient lui étaient versées en personne. Ce comportement a soulevé de nombreux problèmes et, comme nous l'avons évoqué plus haut, ont justifié le déplacement du Ministre de l'Environnement. L'exploitation a été arrêtée dans cette zone et les exploitants ont été expulsés du village après avoir payé une amende d'environ 200000 FCFA $^{16}$.

Ces différents cas nous informent de façon éloquente sur l'évolution des rapports des populations autochtones aux ressources forestières et sur la façon dont le territoire fait désormais l'objet d'appropriations nouvelles. La valeur monétaire de la ressource s'est traduite par une modification des rapports aux exploitants étrangers dont la présence a engendré des tensions entre autochtones en raison, notamment, de la concurrence de leurs intérêts économiques.

\footnotetext{
15 Le 06/04/06, entretien avec T. K, ancien migrant du bois du Mandé, actuellement commerçant de charbon à Bamako dans le marché de Garantigui bougou.

${ }^{16}$ Selon D., le chef d'antenne forestier de Siby.
} 


\section{Conclusion}

Les quelques années d'expérience de la gestion décentralisée ont révélé toutes les incohérences, notamment au niveau financier, de cette politique ainsi que les écarts entre les textes et la réalité. La présence d'étrangers sur les territoires du Mandé a créé un cadre favorable à l'expression de la frustration des populations riveraines vis-à-vis de l'Etat et des autres instances supérieures mais aussi de leur repositionnement par rapport aux pouvoirs de celles-ci, en procédant à des négociations parallèles. Elle a permis l'expression d'une revendication de leur légitimité sur les recettes financières et de leur droit de contrôle sur les ressources et les territoires.

L'analyse des rapports entre populations autochtones et migrants du bois du Mandé a montré que les revendications territoriales sont également la traduction de réelles craintes d'être dominés économiquement par un groupe socio-identitaire étranger aux villages d'accueil. En effet, si la pratique du bûcheronnage peut permettre à des personnes de connaître une véritable ascension sociale, comme le sous-entendent ces craintes, cela ne signifie-t-il pas qu'elle est en train de redynamiser l'économie rurale, à travers la diversification de ses activités, et de restructurer ses espaces?

\section{Bibliographie}

BERTRAND, A. et al., 1992. Etude des filières d'approvisionnement des cinq principales villes du Mali en produits forestiers, Synthèse Tome1, Direction nationale des eaux et forêts, Bamako, $145 \mathrm{p}$.

BRONDEAU, F., 2001, "Evolution de la filière bois énergie et dynamique des formations ligneuses autour de l'Office du Niger. ", Bois et forêts des tropiques, vol. 270(4).

CCL (Cellule Combustible Ligneux), 1998, Schéma directeur d'approvisionnement en bois énergie de Bamako, Bamako (Mali) : Ministère du développement rural et de l'eau, $50 \mathrm{p}$. 
GAUTIER, D., et al., 2004. « Les premiers pas d'un marché rural de bois au Mali : contexte politique et dynamiques locales induites ", L'espace géographique, $\mathrm{n}^{\circ} 4$, pp : 289-305.

GAZULL, L., 2003, Organisation spatiale d'une filière d'approvisionnement en bois énergie d'un grand centre urbain sahélien. Une approche par un modèle d'attraction arborescent, mémoire de DEA de géographie de l'Université de Montpellier III-Paul Valery, $124 \mathrm{p}$.

KEITA, S., 2003, Les statuts des forêts du Mandé (Mali) et leurs modes d'appropriation, Rapport d'étape, ATP-CIRAD/CNRST, 21 p.

MOLLER, M.R., 1998, The changing roles of rural non-agricultural activities in the livelihoods of nigerien peasants, Centre for development research, CDR working paper 98.9 .

NOUVELLET, Y., 2002, Manuel de procédure de mise en place et de suivi des marchés ruraux de bois énergie, Document de la Stratégie Energie Domestique, République du Mali, 43 p.

PEII (Projet Energie II), 1991, Schéma d'approvisionnement en bois-énergie de Niamey, Ministère de l'énergie du Niger - CIRAD, $112 \mathrm{p}$.

RIBOT, J.C., 1990,"Markets, States and environmental policy: The political economy of charcoal in Senegal", PhD Dissertation, University of California.

RIBOT, J.C., 1998, "Theorizing access: forest profits along Senegal's charcoal commodity chain ", Development and Change, 29 : 307-341.

RIBOT, J C., 1995, Le contrôle local des forêts au Burkina Faso, au Mali, au Niger, au Sénégal et en Gambie : examen et critique des nouvelles politiques participatives, Washington : Banque Mondiale, $94 \mathrm{p}$.

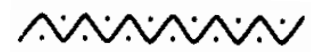

Faty Modj est Docteur de l'Université Paris1 Panthéon-Sorbonne

E-mail : mboja@gmail.com 